\title{
A Clinical Aid for Detecting Skin Cancer: The Triage Amalgamated Dermoscopic Algorithm (TADA)
}

\author{
T. Rogers, MFA, M. L. Marino, MD, S. W. Dusza, DrPH, S. Bajaj, MD, \\ R. P. Usatine, MD, M. A. Marchetti, MD, and A. A. Marghoob, MD
}

Purpose: Family physicians (FPs) frequently evaluate skin lesions but may not have the necessary training to accurately and confidently identify lesions that require skin biopsy or specialist referral. We evaluated the diagnostic performance of a new, simplified dermoscopy algorithm for skin cancer detection.

Methods: In this cross-sectional, observation study, attendees of a dermoscopy course evaluated 50 polarized dermoscopy images of skin lesions ( 27 malignant and 23 benign) using the Triage Amalgamated Dermoscopic Algorithm (TADA). The dermoscopic criteria of TADA include architectural disorder (ie, disorganized or asymmetric distribution of colors and/or structures), starburst pattern, blue-black or gray color, white structures, negative network, ulcer, and vessels. The study occurred after 1 day of basic dermoscopy training. Clinical information related to palpation (ie, firm, dimpling) was provided when relevant.

Results: Of 200 course attendees, 120 (60\%) participated in the study. Participants included 64 (53.3\%) dermatologists and $41(34.2 \%)$ primary care physicians, 19 (46.3\%) of whom were FPs. Fiftytwo (43\%) individuals had no previous dermoscopy training. Overall, the sensitivity and specificity of TADA for malignant skin lesions was $94.8 \%$ and $72.3 \%$, respectively. Previous dermoscopy training and years of dermoscopy experience were not associated with diagnostic sensitivity $(P=.13$ and $P=.05$, respectively) or specificity $(P=.36$ and $P=.21$, respectively). Specialty type was not associated with sensitivity $(P=.37)$ but dermatologists had a higher specificity than nondermatologists $(79 \% \mathrm{v} .72 \%$, $P=.008)$.

Conclusions: After basic instruction, TADA may be a useful dermoscopy algorithm for FPs who examine skin lesions as it has a high sensitivity for detecting skin cancer. (J Am Board Fam Med 2016;29:694-701.)

Keywords: Algorithms; Biopsy; Cross-sectional Studies; Dermoscopy; Palpation; Physicians, Family; Referral and Consultation; Sensitivity and Specificity; Skin Neoplasms; Triage

It has been proposed that the worldwide epidemic of skin cancer $^{1,2}$ be addressed by all physicians involved in the ongoing primary care of patients. ${ }^{3}$

This article was externally peer reviewed.

Submitted 22 February 2016; revised 3 June 2016; accepted 13 June 2016.

From the Dermatology Service, Department of Medicine, Memorial Sloan Kettering Cancer Center, New York, NY (TR, MLM, SWD, SB, MAM, AAM); Department of Family and Community Medicine, University of Texas Health Science Center at San Antonio, San Antonio, TX (RPU).

Funding: This research was funded in part through the NIH/NCI cancer center support grant P30 CA008748.

Conflict of interest: none declared.

Corresponding author: Ashfaq A. Marghoob, MD, Dermatology Service, Department of Medicine, Memorial Sloan Kettering Cancer Center, 16 E. 60th Street, New York, NY 10022 (E-mail: marghooa@mskcc.org).
The current shortage of US dermatologists ${ }^{4,5}$ has heightened the need to involve other medical professionals in skin cancer management. Primary care physicians (PCPs), including family physicians (FPs), regularly see patients with skin concerns who are not being followed by or do not have access to dermatologists. Each of these patient encounters, regardless of its primary purpose, is an opportunity to detect skin cancer. In primary care settings, the clinical assessment of concerning lesions requires the ability to determine whether a biopsy or further evaluation by a specialist is warranted. However, PCPs often lack confidence in their abilities to recognize skin cancer. ${ }^{6}$ The dearth of dermatologic education in medical school curricula and family 
medicine residencies ${ }^{7,8}$ underscores the need for providing PCPs with better tools and training for the management of cutaneous lesions. ${ }^{9}$

Dermoscopy is a noninvasive diagnostic technique that has been shown to enhance the detection of skin cancer compared with naked-eye examination. ${ }^{10-12}$ Although the use of dermoscopy has been gaining popularity among PCPs,${ }^{13}$ training is required for it to become a beneficial tool. ${ }^{14-17}$ To facilitate the use of dermoscopy among nonexperts, several simplified dermoscopic algorithms have been validated. ${ }^{18,19}$ However, these algorithms were designed to detect specific subsets of pigmented skin cancers, primarily pigmented melanoma. This restricts their overall utility given that many basal cell carcinomas, squamous cell carcinomas, and even melanomas are not pigmented. To our knowledge, there are no proven clinical tools available to PCPs for evaluating nonpigmented skin lesions. ${ }^{20}$

The triage amalgamated dermoscopic algorithm (TADA) (Figure 1) was designed for the identification of both pigmented and nonpigmented skin cancers. In our experience, it can be difficult and time consuming to teach beginners the multitude of dermoscopic structures specific to a given malignancy. This is in part due to the poor interobserver concordance for most of these structures. ${ }^{21}$ However, the subjective interpretation of architectural disorder (ie, disorganized or asymmetric distribution of colors and/or structures) has been shown to have high interobserver agreement and discriminatory power for malignancy. ${ }^{21,22}$ TADA harnesses these attributes by prompting users to assess a lesion for architectural disorder, which if present, would suggest the need for a biopsy or specialist referral. However, given that several subtypes of skin cancer, such as nodular, spitzoid, and amelanotic melanoma, can appear as organized and symmetric lesions, TADA includes 6 additional, previously validated, dermoscopic patterns and features (starburst, blue-black or gray color, polarizing white structures, negative network, ulcer, vessels) to help identify these skin cancers. ${ }^{23,24,25-30}$ The presence of any 1 of the 6 additional criteria would also suggest the need for a biopsy or specialist referral.

One major difference between TADA and the other simplified screening algorithms is that TADA requires that commonly encountered and diagnostically unequivocal benign lesions, namely, angioma, dermatofibroma, and seborrheic keratosis, be excluded from the algorithm. In other words, be- fore assessing for the algorithm's malignant criteria, one should first determine whether a lesion is a classic example of 1 of these 3 benign neoplasms. Although in many instances, these lesions can have dermoscopic structures that overlap with malignant lesions, ${ }^{31,32}$ they can, nonetheless, be differentiated based on their overall clinical and dermoscopic patterns. TADA thus asks observers to learn the salient dermoscopic patterns associated with these benign lesions, which, in our personal experience, are easy to teach and learn. In addition, the frequency with which these lesions are encountered in clinical practice allows one to rapidly gain experience in their identification.

The aim of this observational study was to determine the diagnostic accuracy of TADA for common malignant skin lesions (melanoma, basal cell carcinoma, squamous cell carcinoma) when used by individuals with 1 day of dermoscopy training versus those with more extensive training or experience.

\section{Materials and Methods \\ Study Population}

This study was approved by the Memorial Sloan Kettering Institutional Review Board without requirement for written informed consent in accordance with the Helsinki Declaration. The study was open to all attendees of a dermoscopy course and was not restricted by specialty type or prior dermoscopy training or experience.

\section{Selection of Skin Lesions}

The study lesions were selected retrospectively from dermoscopically imaged neoplasms seen by author AAM at Memorial Sloan Kettering Cancer Center in Hauppauge, NY. Sixty-two lesions that were deemed as representative examples of benign and malignant neoplasms by dermoscopists AAM and MAM were selected. Twelve of the 62 lesions were excluded due to suboptimal image quality or lack of polarized dermoscopic images, resulting in 50 study lesions. Melanomas, basal cell carcinomas, and squamous cell carcinomas were histopathologically proven as malignant (27 total: 16 malignant melanoma, including 3 nodular melanoma and 1 amelanotic melanoma, 7 basal cell carcinoma, 4 squamous cell carcinoma). Benign lesions were either biopsied or were known to be stable by comparison with prior images (23 total: 8 nevi, 5 seborrheic keratoses, 5 angioma, 4 dermatofibroma, 1 clear cell acanthoma). Lesions were imaged with 
Figure 1. The Triage Amalgamated Dermoscopic Algorithm (TADA).

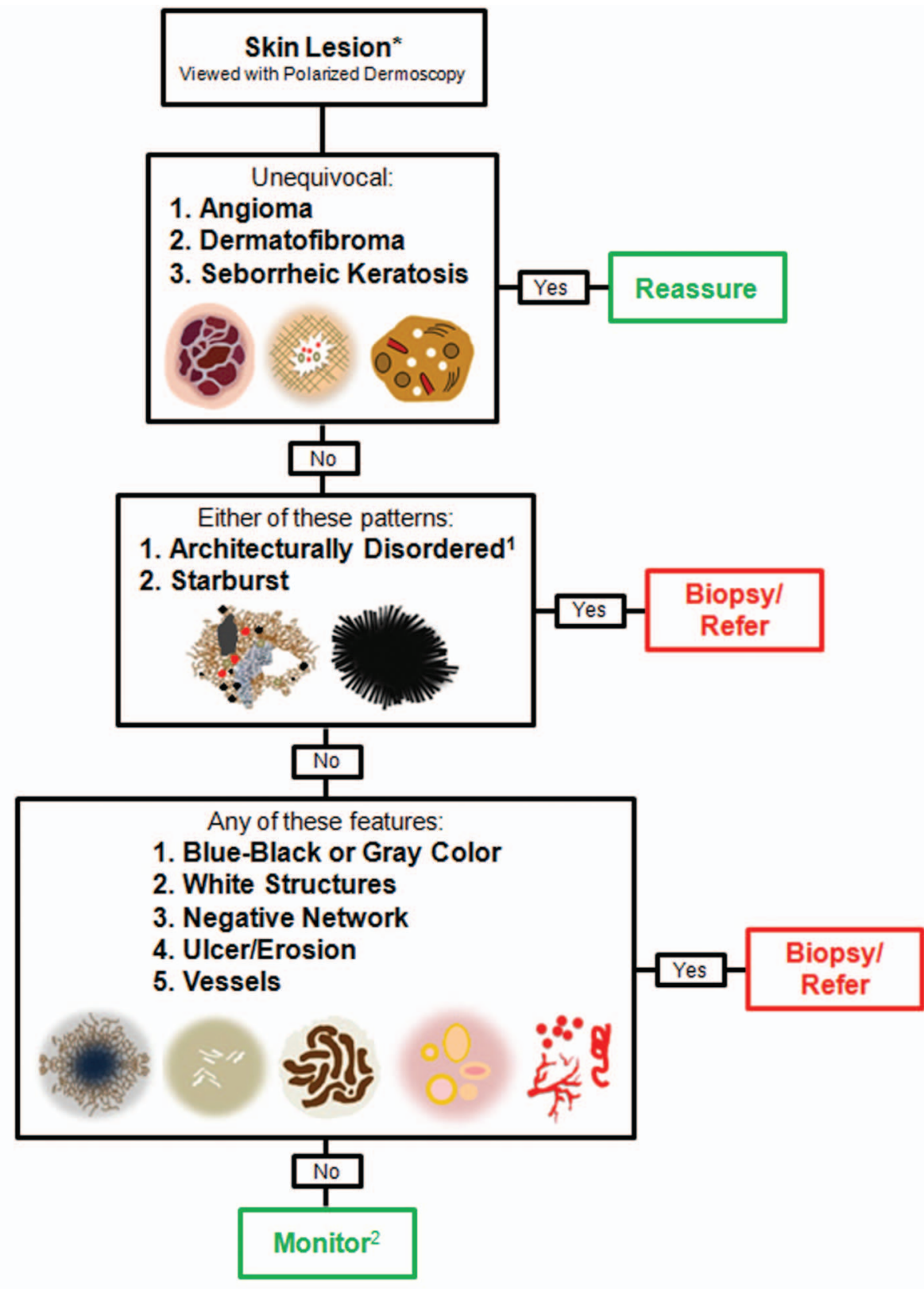

*Exception: Lesions on palms, soles, or nails

'Disorganized or asymmetric distribution of colors and/or structures

aPatient should continue self monitoring and any changes or new symptoms (i.e., itching, bleeding) brought to the attention of their physician

contact polarized dermoscopy at a magnification factor of 10 .

\section{Evaluation of Cases and Data Collection}

The study took place on the second day of a 3-day dermoscopy course, after participants had received 1 day of basic dermoscopy training. This included classroom sessions that covered the published dermoscopic features of common benign and malignant skin lesions, as well as the dermoscopic criteria used in the TADA algorithm. Participants were given quizzes and partook in unknown lesion identification sessions to reinforce the covered topics. Before the study commenced, participants also received a brief tutorial on how to use the TADA algorithm and 
fill out the provided worksheets. Although clinical images of the study lesions were not provided, clinical information related to palpation (ie, rough, firm, dimpling with lateral pressure) was provided when relevant. Dimpling with lateral pressure, for instance, is a common feature of dermatofibroma that can aid in their identification in the clinical setting.

TADA consists of 3 levels, which participants worked through in a stepwise manner for each study lesion. In level 1, participants were asked to determine whether the lesion was an unequivocal example of 1 of 3 benign neoplasms (angioma, dermatofibroma, or seborrheic keratosis). After excluding these 3 , participants then moved to level 2, in which they assessed for the presence of architectural disorder. For lesions deemed as ordered/organized/symmetric, participants then moved to level 3 to evaluate for the remaining criteria. The presence of any 1 criterion from level 2 or 3 was considered to be suggestive of malignancy and indicated the need for a biopsy or specialist referral. After a participant identified any 1 criterion, they were instructed to stop filling out the worksheet and to wait for the next case. If none of the criteria were noted, the participants were led to the management decision of monitoring the lesion. Worksheets were collected and data entered into an electronic database that was used to estimate sensitivities and specificities.

\section{Statistics}

Descriptive statistics and graphical methods were used to assess and describe the characteristics of the study participants, the lesions used in the evaluations, and the individual dermoscopic characteristics of the lesions. A TADA classification variable was created from the responses for each lesion evaluation and coded as dichotomous (TADA positive $=1$; TADA negative $=0$ ). Level of experience in dermoscopy was assessed as a dichotomous variable with those reporting "any dermoscopic training" coded as 1 and no dermoscopic training coded as 0 . In addition, level of experience with dermatology and dermoscopy was assessed as years practicing dermoscopy. To quantify the association for the presence/absence of each TADA feature with malignant status, tabular cross classifications and $\chi^{2}$ statistics were calculated. Measures of diagnostic accuracy along with $95 \%$ confidence intervals (CIs) were estimated using a general estimating equa- tions approach for binary outcomes with the TADA evaluation response as the dependent variable and with the main independent variable being benign/malignant status. Two separate models were independently used to estimate the sensitivity and specificity. Given that study participants contributed multiple observations to the data set, and responses from a given individual have some level of correlation, an exchangeable correlation structure was used in the modeling approach. All analyses were performed with Stata v. 14.0, StataCorp, College Station, TX.

\section{Results}

Of the 200 attendees who were eligible to participate, $120(60 \%)$ completed the study. A majority of the participants were female ( $\mathrm{n}=64 ; 53.3 \%$ ), half were 50 years of age or less ( $\mathrm{n}=59 ; 49.2 \%)$, and a large proportion had medical specialties other than dermatology $(n=49 ; 41 \%)$. Of the latter group, 41 (84\%) described their specialty as family medicine, family medicine/geriatrics, medicine, or medicine/ skin cancer medicine. Over 50 individuals (43.3\%) reported no previous dermoscopy training (Table 1). A total of 5641 lesion evaluations were per-

Table 1. Number of Study Participants Grouped by Profession, Dermoscopy Training, and Dermoscopy Experience

\begin{tabular}{lc}
\hline Characteristic & $\mathrm{n}(\%)$ \\
\hline Profession & \\
Dermatologist & $64(53.3)$ \\
Primary care physician & $41(34.2)$ \\
Other medical specialties & $7(5.8)$ \\
Other profession & $1(0.8)$ \\
Did not answer & $7(5.8)$ \\
Previous dermoscopy training & \\
Yes & $63(52.5)$ \\
No & $52(43.3)$ \\
Did not answer & $5(4.2)$ \\
Previous dermoscopy experience & \\
Yes & $85(70.9)$ \\
No & $28(23.3)$ \\
Did not answer & $7(5.8)$ \\
Years of dermoscopy experience & \\
0 to 1 year & $52(43.3)$ \\
2 to 5 years & $35(29.2)$ \\
6 to 10 years & $18(15.0)$ \\
$>10$ years & $9(7.5)$ \\
Did not answer & $6(5.0)$ \\
\hline
\end{tabular}


formed (3034 malignant and 2607 benign) with an average of 47 evaluations per participant (of a possible 50$)$.

Overall, TADA had a sensitivity of $94.8 \%$ (95\% CI, $93.9 \%-95.5 \%)$ and a specificity of $72.3 \%(95 \%$ CI, $70.5 \%-74.0 \%)$ for all study lesions. Sensitivity estimates for melanoma were $94 \%$. Regarding nonmelanoma skin cancers, participants achieved sensitivities of $95 \%$ for basal cell carcinoma and $96 \%$ for squamous cell. Sensitivity estimates for pigmented versus nonpigmented nonmelanoma skin cancers were $93 \%$ versus $96 \%$ for basal cell carcinoma, respectively, and $95 \%$ versus $97 \%$ for squamous cell carcinoma, respectively. Specificities for benign study lesions were $5 \%$ for clear cell acanthoma, $63 \%$ for nevi, $73 \%$ for angioma, $81 \%$ for seborrheic keratosis, and $93 \%$ for dermatofibroma. The positive predictive value for TADA was $79.9 \%(95 \%$ CI, $78.6 \%-81.2 \%)$ and the negative predictive value was $92.2 \%(95 \%$ CI, $91.0 \%-93.3 \%$ ).

Similar estimates of diagnostic performance were seen when stratified by medical specialty (dermatologists vs nondermatologists), prior dermoscopy training, or experience level with dermoscopy (Table 2). Diagnostic sensitivities achieved by individuals with and without previous dermoscopy training (before the first day of the course) were $95.0 \%$ (95\% CI, 90.9\%99.4\%) versus $93.3 \%$ (95\% CI, 91.5\%-95.1\%), respectively. Participants with prior dermoscopic training also had similar estimates of diagnostic specificity compared with those without, $76.4 \%$ (95\% CI, $66.9 \%-83.1 \%$ ) versus $74.1 \%$ (95\% CI, $70.2 \%-$ $77.5 \%)$, respectively.

\section{Discussion}

More than half of all physician office visits in the US are to PCPs, including FPs. ${ }^{33}$ These physicians are increasingly confronted with the management of skin lesions and are often the first line for skin cancer detection. ${ }^{15,34}$ In one study, $63 \%$ of patients with newly diagnosed melanoma had visited PCPs within the year before their diagnoses. ${ }^{35}$ Despite this, PCPs seem to lack sufficient knowledge and experience for evaluating malignant skin lesions, failing to identify as many as one third of skin cancers. ${ }^{36}$ Lack of training has been a reported barrier preventing these physicians from gaining the confidence and abilities necessary for skin cancer management. Although 54\% of PCPs suggest having sufficient skills for diagnosing skin cancer, ${ }^{37}$ greater than $50 \%$ are requesting further knowledge to aid in the process. ${ }^{38}$

Several studies have demonstrated that dermoscopy can improve PCPs' abilities to accurately identify malignant skin lesions. ${ }^{14,15,17}$ In 2009, the American Academy of Family Physicians held the first dermoscopy course at their Annual Scientific Assembly Meeting. Since then, the demand for this course has increased and is currently being offered at 4 different times during the meeting, up from 3 times the previous year, to accommodate increased interest (published American Academy of Family Physicians program schedules). Although dermoscopy has been a proposed addition to PCPs' skin cancer curricula, ${ }^{16}$ the best dermoscopic method to learn is unknown. The classic approach to dermoscopy relies on users' expert knowledge of myriad and often nuanced dermoscopic structures and pat-

Table 2. Sensitivity and Specificity of TADA Algorithm for Malignant Study Lesions (Melanoma, Basal Cell Carcinoma, Squamous Cell Carcinoma) Achieved by Dermatologists Versus Non-Dermatologists, Participants with Previous Dermoscopy Training Versus Those Without, and Participants with Less Than or Equal to One Year of Dermoscopy Experience Versus Those with Greater than One Year of Experience

\begin{tabular}{|c|c|c|c|c|c|c|c|c|c|c|}
\hline \multirow[b]{2}{*}{ Variable } & \multirow[b]{2}{*}{ Coding } & \multirow{2}{*}{$\begin{array}{l}\text { Participants/ } \\
\text { Observations }\end{array}$} & \multirow[b]{2}{*}{ Sensitivity } & \multicolumn{2}{|c|}{$95 \% \mathrm{CI}$} & \multirow[b]{2}{*}{$P$} & \multirow[b]{2}{*}{ Specificity } & \multicolumn{2}{|c|}{$95 \% \mathrm{CI}$} & \multirow[b]{2}{*}{$P$} \\
\hline & & & & Lower & Upper & & & Lower & Upper & \\
\hline \multirow[t]{2}{*}{ Profession } & Dermatologist & $64 / 2788$ & 0.948 & 0.924 & 0.972 & .374 & 0.785 & 0.702 & 0.844 & .008 \\
\hline & Non-Dermatologist & $49 / 2132$ & 0.937 & 0.919 & 0.955 & & 0.721 & 0.681 & 0.755 & \\
\hline \multirow{2}{*}{$\begin{array}{l}\text { Previous dermoscopy } \\
\text { training }\end{array}$} & Yes & $63 / 2583$ & 0.950 & 0.909 & 0.994 & .13 & 0.764 & 0.669 & 0.831 & .36 \\
\hline & No & $52 / 2132$ & 0.933 & 0.915 & 0.951 & & 0.741 & 0.702 & 0.775 & \\
\hline \multirow{2}{*}{$\begin{array}{l}\text { Years of experience in } \\
\text { dermoscopy }\end{array}$} & $>1$ year & $62 / 2002$ & 0.954 & 0.931 & 0.978 & .05 & 0.773 & 0.683 & 0.837 & .21 \\
\hline & $\leq 1$ year & $52 / 2404$ & 0.931 & 0.913 & 0.950 & & 0.742 & 0.704 & 0.775 & \\
\hline
\end{tabular}

CI, confidence interval; TADA, Triage Amalgamated Dermoscopic Algorithm. 
terns necessary to generate the correct diagnosis. This requires significant training and practice to implement with high accuracy. ${ }^{23}$ To make dermoscopy easier to use for nondermatologists and to aid in its widespread adoption in general practice, several simplified screening algorithms have been introduced. ${ }^{18,19}$ The validation of these algorithms has shown that the inclusion of a select group of dermoscopic features is sufficient to allow inexperienced users to correctly identify a large proportion of skin cancers. However, the fact that these algorithms were designed for the detection of specific subsets of pigmented skin cancers, primarily pigmented melanoma, highlights the need for a new algorithm designed to detect both pigmented and nonpigmented malignancies.

In this study, $34 \%$ of the 120 participants were PCPs and $43 \%$ of the study population had no previous dermoscopy training before the first day of the course. This allowed us to evaluate the potential utility of TADA as an aid for skin cancer evaluations in primary care settings. TADA achieved an overall sensitivity for all skin cancers of $94.8 \%$. This value was not influenced by participants' previous training or experience with dermoscopy, or medical specialty. The fact that training improves the diagnostic accuracy of inexperienced users has been demonstrated in previous studies. ${ }^{14-18}$ However, the amount and type of training provided in these studies has varied considerably, from a 1-hour web-based tutorial plus a 15 lesion training set, ${ }^{18}$ to a 1 -day training course, ${ }^{16}$ to the use of a dermoscopy textbook plus a 245 lesion training set. ${ }^{14}$ Future studies can help to elucidate whether the training modality for TADA can be further streamlined.

The specificity of TADA for all users was $72.3 \%$. Remarkably, participants without dermoscopy training before the study correctly identified around $74 \%$ of benign lesions. These results compare favorably to the values reported from studies evaluating the performance of inexperienced dermoscopists when using other screening algorithms $(32.8 \%-72 \%),{ }^{18,19,39}$ with the added benefit of identifying a greater number and more varied types of malignancies overall. It can thus be concluded that the high sensitivity we observed with TADA would not have been at the expense of an increase in the number of unnecessary biopsies or referrals. In addition, the specificities for the 3 types of benign lesions included in the TADA algorithm ranged from $73.0 \%$ to $93.0 \%$. This finding substantiates our view that it is possible to rather quickly train beginners to accurately identify classic examples of certain common, benign lesions. Although requiring users to gain additional rudimentary dermoscopic knowledge to identify these lesions is arguably a limitation of TADA, it also seemed to strengthen the algorithms indicated by the high specificities achieved for these lesions.

The results of this pilot study suggest that TADA can help inexperienced users who are motivated to learn dermoscopy detect both pigmented and nonpigmented skin cancers with very high sensitivities and specificities. As such, our results may not be generalizable to all PCPs. To make definitive statements about the usefulness of TADA as a clinical tool in primary care, our findings would need confirmation in a larger study with more PCPs evaluating a more diverse and larger sample size. Providing participants with clinical images in addition to dermoscopic images would also more closely correlate with the real-life situation in which the algorithm would be applied. Ideally, this would be achieved with a prospective study performed in a clinical setting. In addition, reader studies, such as this one, are often performed with photographic representations of dermoscopic images. This is not the same as viewing lesions in vivo. A prospective, clinical study would also allow us to account for the logistical aspects of operating a dermoscope. Further, by randomizing participants to various levels and durations of training, we might determine the most efficient teaching method for PCPs. To overcome some of these limitations, we plan to reevaluate the diagnostic accuracy of TADA as a function of training duration and didactic method with a large sample of FPs and family medicine residents.

\section{References}

1. Siegel RL, Miller KD, Jemal A. Cancer statistics, 2015. CA Cancer J Clin 2015;65:5-29.

2. Lomas A, Leonardi-Bee J, Bath-Hextall F. A systematic review of worldwide incidence of nonmelanoma skin cancer. Br J Dermatol 2012;166:1069-80.

3. McDonald CJ. American Cancer Society perspective on the American College of Preventive Medicine's policy statements on skin cancer prevention and screening. CA Cancer J Clin 1998;48:229-31.

4. Kimball AB, Resneck JS, Jr. The US dermatology workforce: A specialty remains in shortage. J Am Acad Dermatol 2008;59:741-5. 
5. Uhlenhake E, Brodell R, Mostow E. The dermatology work force: A focus on urban versus rural wait times. J Am Acad Dermatol 2009;61:17-22.

6. Kirsner RS, Muhkerjee S, Federman DG. Skin cancer screening in primary care: Prevalence and barriers. J Am Acad Dermatol 1999;41:564-6.

7. Wise E, Singh D, Moore M, et al. Rates of skin cancer screening and prevention counseling by US medical residents. Arch Derm 2009;145:1131-6.

8. Moore MM, Geller AC, Zhang Z, et al. Skin cancer examination teaching in US medical education. Arch Derm 2006;142:439-44.

9. Robison S, Kljakovic M, Barry P. Choosing to biopsy or refer suspicious melanocytic lesions in general practice. BMC Fam Pract 2012;13:78.

10. Vestergaard ME, Macaskill P, Holt PE, Menzies SW. Dermoscopy compared with naked eye examination for the diagnosis of primary melanoma: A meta-analysis of studies performed in a clinical setting. Br J Dermatol 2008;159:669-76.

11. Kittler H, Pehamberger H, Wolff K, Binder $M$. Diagnostic accuracy of dermoscopy. Lancet Oncol 2002;3:159-65.

12. Bafounta ML, Beauchet A, Aegerter P, Saiag P. Is dermoscopy (epiluminescence microscopy) useful for the diagnosis of melanoma? Results of a metaanalysis using techniques adapted to the evaluation of diagnostic tests. Arch Derm 2001;137: 1343-50.

13. Argenziano G, Soyer HP. Dermoscopy of pigmented skin lesions-A valuable tool for early diagnosis of melanoma. Lancet Oncol 2001;2:443-9.

14. Menzies SW, Emery J, Staples M, et al. Impact of dermoscopy and short-term sequential digital dermoscopy imaging for the management of pigmented lesions in primary care: A sequential intervention trial. Br J Dermatol 2009;161:1270-7.

15. Westerhoff K, McCarthy WH, Menzies SW. Increase in the sensitivity for melanoma diagnosis by primary care physicians using skin surface microscopy. Br J Dermatol 2000;143:1016-20.

16. Argenziano G, Puig S, Zalaudek I, et al. Dermoscopy improves accuracy of primary care physicians to triage lesions suggestive of skin cancer. J Clin Oncol 2006;24:1877-82.

17. Dolianitis C, Kelly J, Wolfe R, Simpson P. Comparative performance of 4 dermoscopic algorithms by nonexperts for the diagnosis of melanocytic lesions. Arch Derm 2005;141:1008-14.

18. Soyer HP, Argenziano G, Zalaudek I, et al. Threepoint checklist of dermoscopy. A new screening method for early detection of melanoma. Dermatology 2004;208:27-31.

19. Luttrell MJ, McClenahan P, Hofmann-Wellenhof R, Fink-Puches R, Soyer HP. Laypersons' sensitivity for melanoma identification is higher with dermoscopy images than clinical photographs. Br J Dermatol 2012;167:1037-41.
20. Koelink CJ, Jonkman MF, Van Der Meer K, Van Der Heide WK. Examination of skin lesions for cancer: Which clinical decision aids and tools are available in general practice? Eur J Dermatol 2014; 24:297-304.

21. Argenziano G, Soyer HP, Chimenti S, et al. Dermoscopy of pigmented skin lesions: Results of a consensus meeting via the Internet. J Am Acad Dermatol 2003;48:679-93.

22. Henning JS, Dusza SW, Wang SQ, et al. The CASH (color, architecture, symmetry, and homogeneity) algorithm for dermoscopy. J Am Acad Dermatol 2007; 56:45-52.

23. Marghoob AA, Usatine RP, Jaimes N. Dermoscopy for the family physician. Am Fam Physician 2013;88: 441-50.

24. Marghoob A, Braun R, Malvehy J. Atlas of Dermoscopy. 2nd ed. London, UK: Informa Healthcare; 2012.

25. Lallas A, Moscarella E, Longo C, et al. Likelihood of finding melanoma when removing a Spitzoid-looking lesion in patients aged 12 years or older. J Am Acad Dermatol 2015;72:47-53.

26. Argenziano G, Longo C, Cameron A, et al. Blueblack rule: A simple dermoscopic clue to recognize pigmented nodular melanoma. Br J Dermatol 2011; 165:1251-5.

27. Balagula Y, Braun RP, Rabinovitz HS, et al. The significance of crystalline/chrysalis structures in the diagnosis of melanocytic and nonmelanocytic lesions. J Am Acad Dermatol 2012;67:194.e191-e8.

28. Zalaudek I, Kittler H, Hofmann-Wellenhof R, et al. "White" network in Spitz nevi and early melanomas lacking significant pigmentation. J Am Acad Dermatol 2013;69:56-60.

29. Altamura D, Menzies SW, Argenziano G, et al. Dermatoscopy of basal cell carcinoma: Morphologic variability of global and local features and accuracy of diagnosis. J Am Acad Dermatol 2010;62:67-75.

30. Pizzichetta MA, Talamini R, Stanganelli I, et al. Amelanotic/hypomelanotic melanoma: Clinical and dermoscopic features. $\mathrm{Br}$ J Dermatol 2004;150: 1117-24.

31. Braun RP, Rabinovitz HS, Krischer J, et al. Dermoscopy of pigmented seborrheic keratosis: A morphological study. Arch Derm 2002;138:1556-60.

32. Zaballos P, Puig S, Llambrich A, Malvehy J. Dermoscopy of dermatofibromas: A prospective morphological study of 412 cases. Arch Derm 2008;144: 75-83.

33. National Ambulatory Med Care Survey: 2010 Summary Tables. Available from: http://www.cdc.gov/ nchs/data/ahcd/namcs_summary/2010_namcs_web_ tables.pdf. Accessed January 19, 2016.

34. Koelink CJ, Kollen BJ, Groenhof F, van der Meer K, van der Heide WK. Skin lesions suspected of malignancy: An increasing burden on general practice. BMC Fam Pract 2014;15:29. 
35. Geller AC, Koh HK, Miller DR, Clapp RW, Mercer MB, Lew RA. Use of health services before the diagnosis of melanoma: Implications for early detection and screening. J Gen Intern Med 1992;7: 154-7.

36. Pockney P, Primrose J, George S, et al. Recognition of skin malignancy by general practitioners: Observational study using data from a populationbased randomised controlled trial. $\mathrm{Br} \mathrm{J}$ Cancer 2009; 100:24-7.

37. Oliveria SA, Heneghan MK, Cushman LF, Ughetta EA, Halpern AC. Skin cancer screening by dermatologists, family practitioners, and internists: Barriers and facilitating factors. Arch Derm 2011;147: 39-44.

38. van Rijsingen MC, van Bon B, van der Wilt GJ, Lagro-Janssen AL, Gerritsen MJ. The current and future role of general practitioners in skin cancer care: An assessment of 268 general practitioners. Br J Dermatol 2014;170:1366-8.

39. Zalaudek I, Argenziano G, Soyer HP, et al. Threepoint checklist of dermoscopy: An open internet study. Br J Dermatol 2006;154:431-7. 